with flow diversion in matched controls who were treated with Clopidogrel. We compare P2Y12 reaction unit (PRU) levels at sets of time point pre, peri and post-operatively, to measure response to Prasugrel versus Clopidogrel. We also evaluate if sub-optimal responses to Prasugrel or Clopidogrel resulted in delay of patient care. Early findings suggest that treatment with Prasugrel results in faster time to therapeutic PRU levels and more consistent PRU levels as compared to Clopidogrel resulting in less delay of care and operative rescheduling.

Disclosures E. Milosavljevic: None. C. McKinney: None. D. Hoss: None.

\section{E-225 MANAGEMENT, COMPLICATIONS AND NEUROLOGICAL OUTCOMES OF ANEURYSMAL SUBARACHNOID HAEMORRHAGE IN ELDERLY PATIENTS}

P Cheng ${ }^{*}$, H Simms, A Abouharb. Department of Neurosurgery, Royal Victoria Hospital, Belfast, UK

\subsection{6/neurintsurg-2020-SNIS.256}

Objective To study the management of aneurysmal subarachnoid haemorrhage and compare neurological outcomes in different elderly age groups.

Design Retrospective cohort study

Methods Patients with aneurysmal subarachnoid haemorrhage (aSAH) admitted to Royal Victoria Hospital Belfast between 2015 to 2019 were separated into different age groups, all patients above age of 70 were enrolled to this study. Study population was further divided into 3 sub-groups, age 70 74 , age $75-79$ and age $>80$. Patient characteristics and clinical courses were compared, including underlying co-morbidities, WFNS grade of aSAH, intervention received, complications and long-term neurological outcomes in follow up clinics.

Results A total of 54 patients were included, with 29 in group I (age $70-74$ ), 20 in group II (age 75 - 79) and 5 in group III (age >80). Despite patients in group III presented with only WFNS grade $1 \& 2$ aSAH, mortality increased exponentially with age, from $10.3 \%$ to $15 \%$ to $40 \%$ across 3 subgroups. There was also a linear increase in average length of stay from 21 days to 24 days. 23 patients (79.3\%) developed complications in group I and 15 patients $(75 \%)$ in group II. 3 patients $(60 \%)$ in group III developed complications and the other 2 patients within same sub-group did not survive. Most common complications were hydrocephalus and hospital acquired infections, $44.4 \%$ of patients developed each condition respectively.

Conclusion Our study suggests patients with age $>80$ had less favourable neurological outcomes despite having good grade aSAH at presentation and received similar intervention, when compared to other age groups. Average length of stay in hospital also increased with age. Similar complication rates were noticed in all age groups. Comparing our data with other neurosurgical units in the United Kingdom and Ireland will provide further information in managing elderly aSAH patients and facilitate risk stratification when considering those patients for intervention.

Disclosures P. Cheng: None. H. Simms: None. A. Abouharb: None.

\section{E-226 FLOW DIVERSION IN ANTERIOR COMMUNICATING ARTERY ANEURYSMS}

K Dakay*, G Kaur, F Al-Mufti, C Gandhi, J Santarelli. Neurosurgery, Westchester Medical Center, Valhalla, NY

\subsection{6/neurintsurg-2020-SNIS.257}

Introduction Anterior communicating artery (ACOM) aneurysms are the most common location encountered in clinical practice, but can be often be challenging to treat. Flow diversion has emerged as an alternative modality for treatment in patients with ACOM aneurysms, although published reports are presently limited.

Materials and Methods We retrospectively evaluated all patients treated at our center from May 2017-February 2020 who underwent flow diversion for an ACOM aneurysm. We defined ACOM aneurysms as any aneurysm involving the ACOM itself, or at the junction of the anterior cerebral artery with the ACOM (A1/A2); both ruptured and unruptured aneurysms were included. We collected baseline clinical and demographic data and angiographic data from both pre- and post-treatment imaging when available; the primary measure was complete occlusion of the aneurysm on follow-up angiogram, defined as no residual filling of either neck or dome of the aneurysm. Parent vessel stenosis and peri-procedural complications were collected as secondary outcomes.

Patients underwent flow diversion with a Pipeline stent under general anesthesia; the procedure was performed under transfemoral access with a $8 \mathrm{Fr} 45 \mathrm{~cm}$ sheath using the Cook shuttle (Cook Medical) as the guide catheter, with the Phenom Plus (ev3) as the intermediate and the Phenom 027 microcatheter (ev3) for distal access. The Navien (Medtronic) intermediate catheter was used in lieu of the Phenom Plus in a minority of cases. A single flow diverting stent was placed in the dominant ACA spanning from the A2 segment extending into the A1 segment in a majority of cases; one case utilized two stents for an H-pipe technique.

Results 19 patients underwent a total of 20 flow diversion procedures for ACOM aneurysm within the study period; median age was 57 and $12(63 \%)$ were male. 12 patients presented with subarachnoid hemorrhage, 3 presented with headache and 4 were found incidentally on workup for an unrelated condition. The median aneurysm size was $4.5 \mathrm{~mm}$, with a range from 2-15 $\mathrm{mm}$. Most patients had significant anterior cerebral artery asymmetry (17; 89\%); a minority of patients exhibited co-dominance. There was one peri-operative access site complication requiring surgical intervention; no cases of intraoperative thrombosis, intracerebral hemorrhage, or stroke were observed. 13 patients underwent follow-up angiogram, of which 8 patients exhibited complete elimination of the aneurysm and 12/13 with protection of the dome. One patient underwent re-treatment with a second flow-diversion procedure.

Conclusion In our experience, flow diversion of ACOM aneurysms is safe and effective, either as a primary treatment modality in an unruptured aneurysm or as a complement to initial coil protection of a ruptured aneurysm. It is best suited for aneurysms asymmetrically located at or near the A1-A2 junction. Further followup and additional studies are needed to confirm these results.

Disclosures K. Dakay: None. G. Kaur: None. F. Al-Mufti: None. C. Gandhi: None. J. Santarelli: None. 